\title{
Underlying physiological and biomechanical mechanisms related to postural control of Parkour practitioners: a pilot study
}

\author{
ANDRÉ F. V. VENEROSO1 | PATRICK W. SEGUNDO' | DANIELA GODOI' \\ 1 Dinâmica - Motor Behavior Laboratory, Department of Physical Education, Federal University of São Carlos, São Carlos, SP, Brazil. \\ Correspondence to: Daniela Godoi, Dinâmica - Motor Behavior Laboratory, Department of Physical Education, Federal University of São Carlos - Rod. Washington Luís, \\ km 235 - SP-310, CEP 13565-905, São Carlos, SP, Brazil. \\ email: danielagodoij@ufscar.br \\ https://doi.org/10.20338/bjmb.v15i2.207
}

\begin{abstract}
HIGHLIGHTS
- Tracers show a lower amount of sway than physically active subjects.

- Tracers show a lower amplitude of the torque required for stabilization than physically active subjects.

- Tracers show a higher degree of postural stability than physically active subjects.

- The use of sensory inputs to control balance is different in tracers.

- The underlying physiological and biomechanical mechanisms related to postural control are different in tracers.
\end{abstract}

$\begin{array}{ll}\text { ABBREVIATIONS } \\ \text { ANOVAs } & \text { Analyses of variance } \\ \text { AP } & \text { Anterior-posterior } \\ \text { CoP } & \text { Center of pressure } \\ \text { MD } & \text { Mean distance between } \\ & \text { successive peaks } \\ \text { ML } & \text { Medial-lateral } \\ \text { MP } & \text { Mean value of the peaks } \\ \text { MT } & \text { Mean time interval between } \\ & \text { successive peaks } \\ \text { RMS } & \text { Root mean square } \\ \text { SDC } & \text { Sway Density Curve }\end{array}$

PUBLICATION DATA

Received 19102020

Accepted 05122020

Published 01062021

\begin{abstract}
BACKGROUND: Parkour can be seen as a sport, an art, a philosophy, a state of mind, an art of living. Practitioners (known as "tracers") have to overcome obstacles in their path by adapting their movements to the given environment to reach somewhere or something or to escape from someone or something. However, the knowledge about the underlying mechanisms related to postural control in tracers is still lacking.

AIM: To examine the postural control in tracers using global, structural, and spectral stabilometric descriptors. METHOD: Five tracers and five controls, all-male, stood upright for 30 seconds, under different conditions of vision (open or closed eyes), surface (soft or rigid), and base of support (bipedal, semi-tandem, or Parkour stance). RESULTS: In more challenging conditions, the tracers compared to controls, showed a lower amount of sway, needed less postural commands, and used sensory information to control balance differently.

CONCLUSION: Tracers have better postural control than controls. Moreover, although current findings are based on data from a small number of subjects, the results suggest that these differences between groups are related to different underlying physiological and biomechanical mechanisms related to postural control.
\end{abstract}

KEYWORDS: Tracers | Postural Control | Sensory Information | Control Mechanisms

\section{INTRODUCTION}

Postural control involves not only balance but also the ability to assume and maintain a desired orientation; so, every movement involves postural control. ${ }^{1}$ Therefore, the postural control system's accurate functioning allows us to interact with the environment properly. However, for this to occur, it is necessary to get information about the environment, which is possible from multiple sources of sensory inputs.

Sensory information comes from, mainly, the visual, vestibular, and somatosensory sensory systems. ${ }^{2}$ Nevertheless, sensory integration for postural control is not merely a summation of inputs from different sensory systems, but a non-linear process named

\begin{tabular}{l|l|l|l|ll}
\hline $\begin{array}{l}\text { Veneroso, } \\
\text { Segundo, Godoi }\end{array}$ & 2021 & VOL.15 & N.2 & https://doi.org/10.20338/bjmb.v15i2.207
\end{tabular}


sensory reweighting. ${ }^{3,4}$ In this way, whenever environmental or central nervous system conditions change, sensory inputs must be dynamically reweighed to optimize the control of postural stability. ${ }^{3}$

This dynamic sensory reweighting process allows us to properly perceive the environment and then appropriately act in that environment. Thus, the action is influenced by the perceived environment, and that action may influence the perceived environment. ${ }^{5}$ In Gibson's words, action leads to the detection of information, and information plays a vital role in controlling the action. ${ }^{6}$ So, it can be said that people perceive to move and move to perceive ${ }^{7}$.This mutual dependency of action and perception is designated as the formation of an action-perception pattern ${ }^{5}$ or cycle ${ }^{1}$; that is, an action-perception coupling. ${ }^{7}$

Interestingly, postural control functioning is not ready at the beginning of life; on the contrary, it changes throughout life. As a result, the ability to select and use sensory information for the appropriate and consistent functioning of the postural control system according to the environmental demands depends on age, 4,8,9, and practice. ${ }^{10,11}$

For this reason, several studies have examined the sport training effects on postural control11,12,13,14 in experimental conditions that manipulated vision ${ }^{11,12,14}$ and somatosensory ${ }^{14}$ information. And, among athletic training, Parkour emerge as an interesting option.

Parkour is derived from the French word parcourt meaning "obstacle course," and was created in Paris's suburbs by David Belle and Sébastien Foucan. ${ }^{15}$ It is defined as an art allowing to pass any obstacle to go from one point of space to another with the possibilities offered by the human body. ${ }^{16}$ Thus, Parkour involves practitioners (called "tracers") training to overcome obstacles in their path by adapting their movements to the given environment to reach somewhere or something or escaping from someone or something. ${ }^{17}$ There are several specific Parkour movements, and these movements vary according to environmental conditions. In general, it can be mentioned vaults (movements that involves overcoming an obstacle by climbing, jumping, or diving over an obstacle while using feet, hands, or not touching it at all), precision jumps (jump from or jump to a specific point from a stationary position), wall runs (horizontal or vertical runs, used to get over a wall too high), and "cat leaps" or arm leap (jump used to land on a ledge, a wall, or a fence). ${ }^{18}$

Due to the rapid growth of this activity worldwide ${ }^{16,19}$ and the fact that this activity had been recognized as a discipline by the International Gymnastics Federation, studies have been conducted to understand tracer's performance better. Most studies have been interested in injuries caused by this activity, ${ }^{15}$ sociocultural aspects, ${ }^{16,19}$ strength and power performance, ${ }^{20}$ and biomechanics characteristics of landing. ${ }^{17}$

However, few studies have been carried out to evaluate the effect of such practice on postural control. To the best of our knowledge, there are only two studies that investigated postural control of tracers. One pilot study ${ }^{18}$ that investigated postural control during the maintenance of a standing upright position, and another study ${ }^{21}$ that investigated postural control during Parkour landing. Nonetheless, in these studies, postural stability was described by linear descriptors (global descriptors such as the area of the center of pressure). Thus, it was possible to describe the amount of sway but not the dynamics that regulate balance control.

To describe and understand these underlying physiological and biomechanical mechanisms related to postural control, more robust analyses should have been employed, 


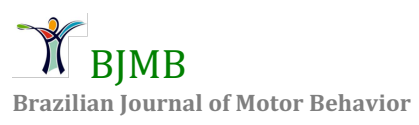

Research Article

including spectral and structural stabilometric descriptors. In this sense, it can be mentioned some analyses such as the Sway Density Curve (SDC) analysis of the center of pressure $(\mathrm{CoP}),{ }^{22}$ which provide information related to the process of generating sequences of postural commands, $23,24,25$ or spectral analysis in different bands of frequency, which assumes that different frequency bands are related to control based on different sources of sensory inputs ${ }^{12,13}$ and, therefore, provides information about the use of different sources of sensory inputs by the postural control system.

Thereby, overall knowledge about the underlying physiological and biomechanical mechanisms related to postural control in tracers is still lacking. Hence, there is a need to gain insight into the dynamics that regulate these practitioners' balance control.

Therefore, the present pilot study aimed to examine the postural control in tracers using global, structural, and spectral stabilometric descriptors. It was hypothesized that tracers (a) would show a lower amount of sway (global descriptors), (b) would need less postural commands to control balance (structural descriptors), and (c) would use sensory inputs in a different way (spectral descriptors), when compared to control subjects with no prior experience in Parkour.

\section{METHODS}

\section{Participants}

Ten male participants (tracers and controls) volunteered for this study. The Tracer group ( $n=5$; mean age: $21.40 \pm 2.70$ years) had participated in Parkour training for at least one year. They trained around two times per week, and each training session lasted between 70 and 80 minutes, divided into four parts: 1) 5-10 min of warm-up activities; 2) 25-30 min of specific Parkour movements, such as vaulting, climbing, precision jump, quadrupedal movement, wall run; 3) 15-20 min of path activities where the goal is to go from one point of space to another by using specific Parkour moves to overcome obstacles in their path by adapting their movements to the given environment; and 4) 15-20 min of challenging activities which included tasks (either technical Parkour movements or path activities) more difficult than those practiced earlier in that session training.

The Control group ( $n=5$; mean age: $23.80 \pm 2.95$ ) had been involved in other physical activities for at least one year, and had no prior experience in Parkour training. All participants gave their informed consent after the procedures were fully explained. The study was conducted following the Declaration of Helsinki and approved by the Institutional Review Board of the Federal University of São Carlos (3.021.618/2018).

\section{Procedures}

Participants were asked to maintain an upright position barefoot on a force platform (Advance Mechanical Technology Inc.- AMTI - AccuGait) with their arms alongside their body, look straight ahead, and maintain their position for 30 seconds. All participants underwent different conditions of vision (open or closed eyes), surface (soft or rigid), and base of support (bipedal stance, semi-tandem stance, or Parkour landing stance) (Figure 1). These conditions were manipulated to generate different levels of challenge to the postural control system, which is considered a classic strategy to unveil the postural control functioning. ${ }^{3}$ 


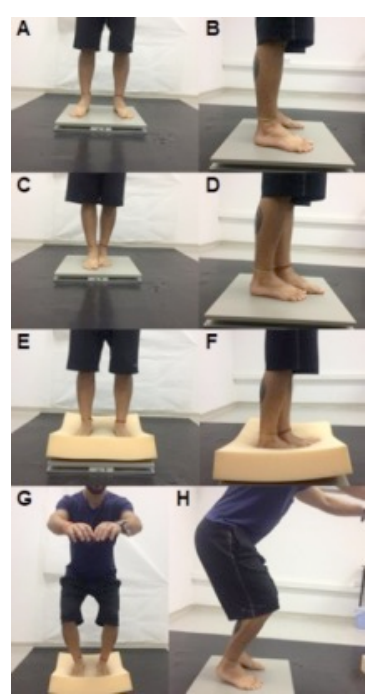

Figure 1. Pictures of the experimental conditions showing the conditions of rigid surface (A-D), soft surface $(\mathrm{E}-\mathrm{G})$, bipedal stance $(\mathrm{A}, \mathrm{B}, \mathrm{E})$, semi-tandem stance $(\mathrm{C}, \mathrm{D}, \mathrm{F})$, and Parkour stance $(\mathrm{G}-\mathrm{H})$.

Under the open eyes condition, participants fixated on a target (a filled circle in black with $5 \mathrm{~cm}$ of diameter in white background) placed at eye level and 1.0 meter in front of them. Under the soft surface condition, a 10 -cm thick foam block (density $=35.0 \mathrm{~kg} / \mathrm{m}^{3}$; elastic modulus $=50,000 \mathrm{~N} / \mathrm{m}^{2}$ ) was placed on force platform (Figure 1E-G). Under the bipedal stance condition, participants stood with their feet parallel to each other and approximately shoulder-width apart (Figure 1A-B, E). Under the semi-tandem stance, participants stood with the foot's hallux positioned behind, touching the medial edge of the calcaneus of the other foot (Figure 1C-D, F). Under the Parkour stance condition, participants stood on the forefoot, bending the knees without varus or valgus movement of the knees and using the arms to keep balance (Figure $1 \mathrm{G}-\mathrm{H}$ ).

These conditions of vision, surface, and base of support resulted in twelve experimental conditions: (1) Eyes open, rigid surface and bipedal stance; (2) Eyes open, rigid surface and semi-tandem stance; (3) Eyes open, soft surface and bipedal stance; (4) Eyes open, soft surface and semi-tandem stance; (5) Eyes closed, rigid surface and bipedal stance; (6) Eyes closed, rigid surface and semi-tandem stance; (7) Eyes closed, soft surface and bipedal stance; (8) Eyes closed, soft surface and semi-tandem stance; (9) Eyes open, rigid surface and Parkour stance; (10) Eyes open, soft surface and Parkour stance; (11) Eyes closed, rigid surface and Parkour stance; and (12) Eyes closed, soft surface and Parkour stance.

Two trials were performed in each condition, totalizing 24 trials per participant, with the order of conditions defined randomly. A resting period ranging from 10 to 20 seconds was provided after each trial.

\section{Data analysis}

Signals from the force platform were recorded at a frequency of $200 \mathrm{~Hz}$ and filtered using a $2^{\text {nd }}$ order zero-lag low-pass Butterworth filter, with a cut-off frequency of $12.5 \mathrm{~Hz}$. To 
compute features from the CoP displacements, global, spectral, and structural analyses were employed in the anterior-posterior (AP) and medial-lateral (ML) directions, except in the case of the structural analysis.

The global analyses were used to describe postural control performance. The descriptors selected were: root mean square (RMS), mean velocity, and amplitude of the CoP. RMS was calculated by subtracting the average position from the original signal (using a de-trending operation) and then by calculating the standard deviation of the AP and ML $\mathrm{CoP}$ trajectories (in centimeters). Mean velocity is the average velocity of the CoP displacement in the AP and ML directions (in centimeters per second). Amplitude is the absolute value of the difference between the smallest and largest values in the AP and ML time series (in centimeters).

The structural analysis chosen was the SDC, which is defined as a time-dependent curve that counts the number of consecutive CoP samples falling inside a circle with a 2.5$\mathrm{mm}$ radius for each instant of time.22,23 The peaks of the resulting curve represent instants of momentary postural stabilization, while the valleys are related to shifts between stabilization events. ${ }^{25}$ The SDC descriptors selected for analysis in the present study were: MP, the mean value of the peaks (in seconds), which is an estimate of the degree of postural stability; MD, the mean distance between successive peaks (in centimeters), which corresponds to the amplitude of torque required for stabilization; MT, the mean time interval between successive peaks (in seconds), which is related to the rate of torque production. ${ }^{23,25}$ These descriptors describe the process of generating sequences of postural commands. ${ }^{22}$ Therefore, this analysis may reveal more clearly physiological and biomechanical mechanisms related to postural control.

The spectral analyses were used to infer the use of sensory information by the postural control system. The descriptor selected was the mean power spectrum of the CoP. The power spectrum of the CoP displacement obtained in each trial was estimated using Welch's method. The mean power spectrum was calculated in the intervals $0.0-0.3,0.3-1.0$, and $1.0-3.0 \mathrm{~Hz} \cdot{ }^{12,26}$ This analysis assumes that the low-frequency band is related to visual control. The middle-frequency band is related to vestibular and somatosensory information, and the high-frequency band is related to proprioceptive control and muscle activity ${ }^{12,13,26}$. Thus, this analysis may provide relevant information about the physiological mechanisms related to postural control.

All variables were calculated using a custom-designed Matlab code.

\section{Statistical Analysis}

The average of two trials in each experimental condition was calculated for each participant. Data were normalized by each participants' body weight and height. ${ }^{27}$ The Normality and the homogeneity of the data were verified by the Shapiro-Wilk test and the Levene test. Then, analyses of variance (ANOVAs) with group (control and Parkour) as factor were conducted for each experimental condition. Dependent measures were RMS, mean velocity, MD, MT, MP, mean power in the intervals $0.0-0.3,0.3-1.0$, and $1.0-3.0 \mathrm{~Hz}$ (low-, middle-, and high-frequency bands). The effect size (ES) was calculated for all dependent variables and classified as small $(<0.2)$, moderate $(0.2-0.79)$, and large $(>0.8) .{ }^{28}$ All the analyses were performed utilizing IBM SPSS software and a-level at 0.05 . 


\section{RESULTS}

Results revealed significant differences between tracers and controls in global, structural, and spectral descriptors under some experimental conditions.

\section{Global Descriptors}

ANOVAs revealed that under experimental condition 11, the tracers showed lower values for $R M S$ in $M L$ direction $[F(1,9)=8.776, p=0.018$, ES: 0.52 (moderate)], mean velocity in AP direction $[F(1,9)=6.999, p=0.029$, $E S: 0.47$ (moderate)], and amplitude in ML direction $[F(1,9)=5.910, p=0.041$, ES: 0.42 (moderate)] than controls (Figure 2).
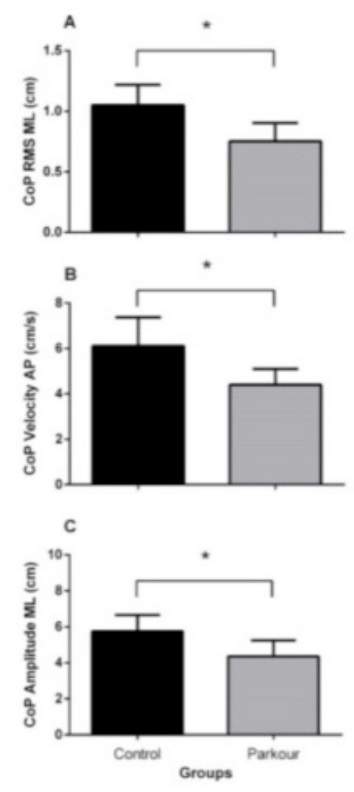

Figure 2. RMS in ML direction (A), Mean Velocity in AP direction (B), and Amplitude in ML direction (C) values for both groups in the experimental condition 11 (Eyes closed, rigid surface and Parkour stance). *Significantly different, $p<0.05$. Values presented as mean and standard deviation.

Moreover, ANOVAs revealed that under experimental condition 12, the tracers showed lower values for mean velocity in $M L$ direction $[F(1,9)=6.549, p=0.034$, ES: 0.45 (moderate)], and amplitude in $\mathrm{ML}$ direction $[F(1,9)=5.509, p=0.047$, ES: 0.41 (moderate)] than controls (Figure 3). 


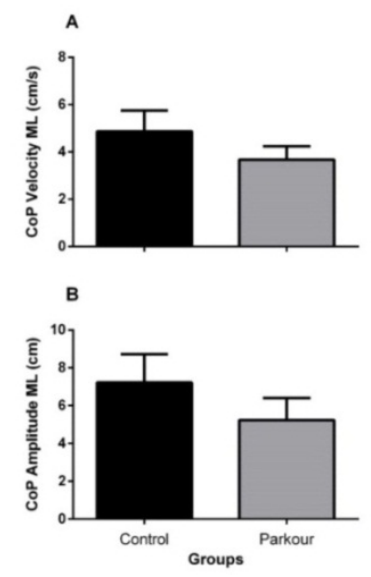

Figure 3. Mean Velocity in ML direction (A), and Amplitude in ML direction (B) values for both groups in the experimental condition 12 (Eyes closed, soft surface and Parkour stance). *Significantly different, $p<0.05$. Values presented as mean and standard deviation.

\section{Structural Descriptors}

ANOVAs revealed that the tracers showed lower values for MD than controls under experimental conditions $2[F(1,9)=6.234, p=0.037$, ES: 0.44 (moderate)], $6[F(1,9)=5.923$, $\mathrm{p}=0.041, \mathrm{ES}: 0.43$ (moderate)], and $12[\mathrm{~F}(1,9)=6.309, \mathrm{p}=0.036, \mathrm{ES}: 0.44$ (moderate)] (Figure 4).
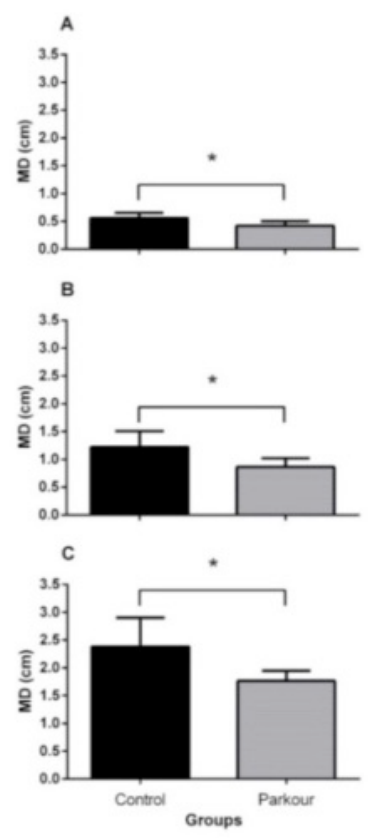

Figure 4. MD values for both groups in the experimental conditions 2 (Eyes open, rigid surface and semitandem stance) (A), 6 (Eyes closed, rigid surface and semi-tandem stance) (B), and 12 (Eyes closed, soft surface and Parkour stance) $(C)$. ${ }^{*}$ Significantly different, $p<0.05$. Values presented as mean and standard deviation. 
Furthermore, ANOVAs revealed that the tracers showed higher values for MP than controls under experimental conditions $11[F(1,30)=5.858, p=0.042$, ES: 0.40 (moderate)], and $12[F(1,30)=5.393, p=0.049$, ES: 0.43 (moderate)] (Figure 5).
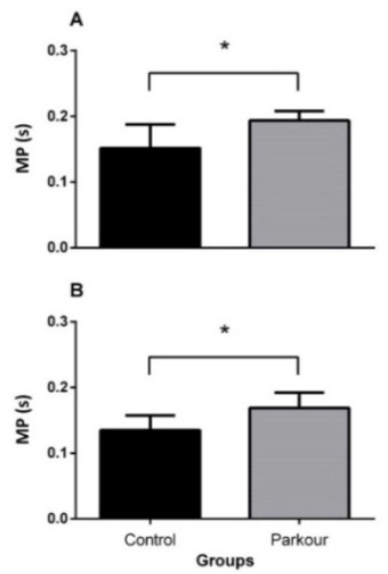

Figure 5. MP values for both groups in the experimental conditions 11 (Eyes closed, rigid surface and Parkour stance) (A), and 12 (Eyes closed, soft surface and Parkour stance) (B). ${ }^{*}$ Significantly different, $p<0.05$. Values presented as mean and standard deviation.

\section{Spectral Descriptors}

ANOVAs revealed that under experimental condition 6 , the tracers showed lower values for mean power in ML direction at the middle-frequency band $[F(1,9)=5.961, p=0.04$, ES: 0.43 (moderate)] than controls; under experimental condition 11, the tracers showed lower values for mean power in AP direction at the high-frequency band $[F(1,9)=8.607$, $p=0.019$, ES: 0.52 (moderate)], and for mean power in ML direction at the low$[F(1,9)=14.884, p=0.003, E S: 0.69$ (moderate)], middle- $[F(1,9)=9.355, p=0.016$, ES: 0.54 (moderate)], and high-frequency $[F(1,9)=7.517, p=0.025, E S: 0.47$ (moderate)] bands than controls; under experimental condition 12, the tracers showed lower values for mean power in AP direction at the high-frequency band $[F(1,9)=6.382, p=0.035$, ES: 0.44 (moderate)], and for mean power in $\mathrm{ML}$ direction at the high-frequency band $[F(1,9)=5.662, p=0.045$, $E S$ : 0.43 (moderate)] than controls (Figure 6). 

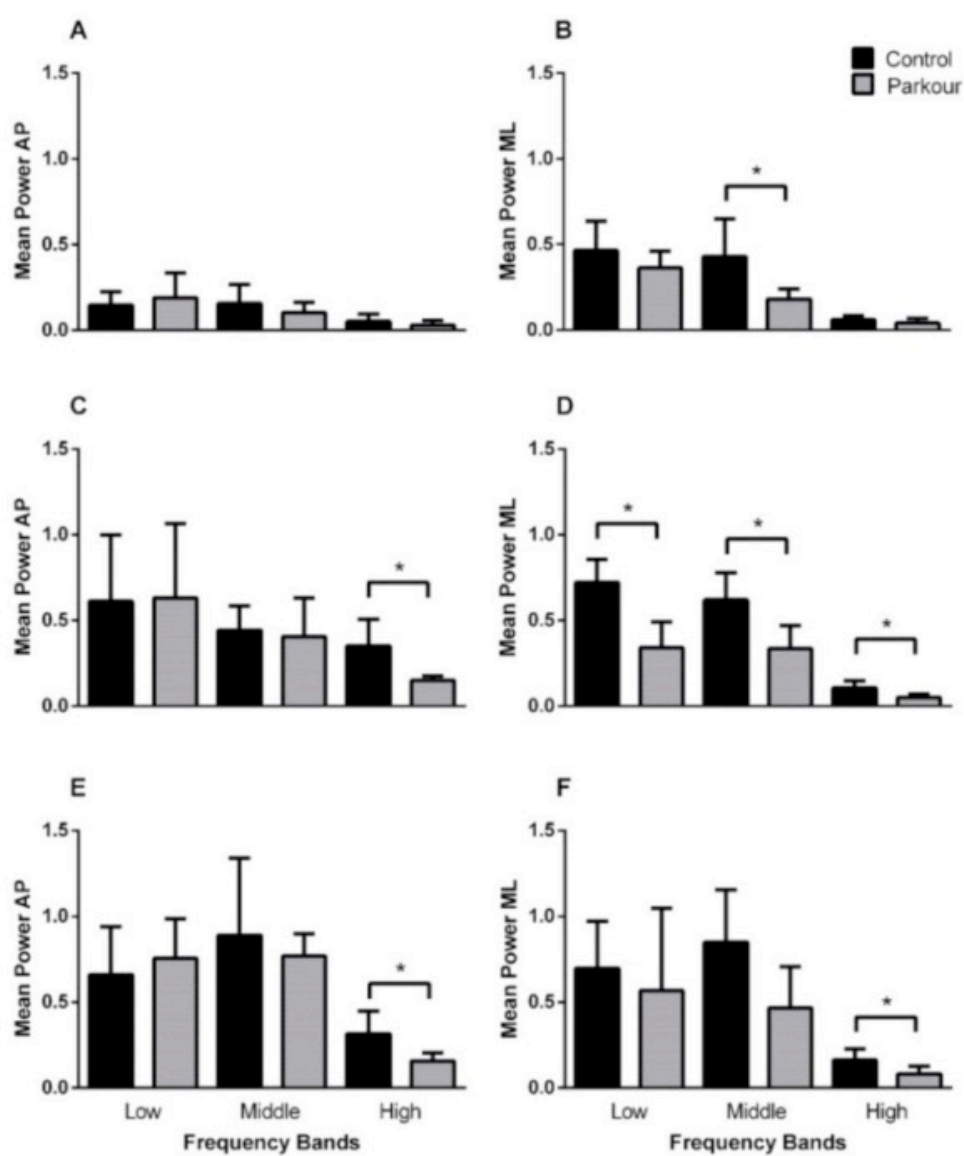

Figure 6. Mean power for both groups in the AP $(A, C, E)$ and $M L(B, D, F)$ direction at different frequency bands under experimental conditions 6 (Eyes closed, rigid surface and semi-tandem stance) (A-B), 11 (Eyes closed, rigid surface and Parkour stance) (C-D), and 12 (Eyes closed, soft surface and Parkour stance) (E-F). *Significantly different, $p<0.05$. Values presented as mean and standard deviation.

\section{DISCUSSION}

The present pilot study aimed to examine the postural control in tracers using global, spectral, and structural stabilometric descriptors. The main results showed that tracers (a) displayed lower variability, velocity, and amplitude of the CoP displacement than controls, (b) needed less postural commands to control balance when compared to controls, and (c) used sensory inputs differently when compared to controls. These differences between tracers and controls were not observed in all experimental conditions, only in more challenging conditions such as semi-tandem and Parkour stances, without vision information, and under the soft surface. Thus, the hypotheses were partially confirmed. These results will be discussed below.

\section{Amount of Body Sway}

Regarding the global descriptors, tracers showed lower variability, velocity and amplitude of CoP displacement than controls. However, these differences were observed 
only during Parkour stance with eyes closed and rigid (Figure 2) and soft (Figure 3) surfaces. Thus, the significant differences in global descriptors were found only in a specific posture (Parkour stance) and in a more challenging condition (without vision information).

These results are similar to other studies that used global descriptors and found that trained subjects ${ }^{11,18}$ showed better postural control only in the trained postures. Asseman et al. ${ }^{11}$ found that expertise in gymnastics only affected postural stability when performing postures similar to those that have been trained; on the contrary, in an unspecific posture (i.e. bipedal stance), gymnasts had similar postural performance compared to untrained subjects. Similarly, Jabnoun et al. ${ }^{18}$ observed differences between tracers and recreationally active subjects only in more challenging postural conditions.

\section{Postural Commands and Use of Sensory Information}

To the best of our knowledge this is the first study to use SDC and spectral descriptors to investigate the postural control in tracers.

Concerning SDC descriptors, tracers showed lower MD values than controls when standing on semi-tandem stance and rigid surface in both vision conditions, and when standing on Parkour stance with closed eyes in the rigid surface. Moreover, tracers showed higher MP values than controls when standing on Parkour stance with closed eyes in both surface conditions.

The parameters extracted from the SDC analysis can be related to both anticipatory and postural stability control. ${ }^{26}$ Thus, the SDC attempts to identify the motor control actions hidden in the CoP signals. ${ }^{24}$ In this sense, the higher MP values observed for tracers (Figure 5 ) reflects the higher degree of postural stability. ${ }^{23,25}$ In addition, considering that the SDC peaks are related to the active torque component, ${ }^{23,26}$ the lower MD values observed for the tracers (Figure 4) indicates lower amplitudes of the torque required for stabilization. ${ }^{23,25}$

Although there were no studies about Parkour with these descriptors, lower MP values, and higher MD values have been associated with poor postural stability. ${ }^{22,24}$ Thus, our results indicate that tracers have better postural control than controls.

Regarding the spectral analysis, significant differences between tracers and controls were found (Figure 6). Given that the information provided by each sensory modality is unique, and each class of receptor operates optimally within a specific range of frequency and amplitude of body motion, ${ }^{3}$ the spectral analysis in different bands of frequency can provide relevant information about distinct sources of sensory inputs used by subjects to control balance.

This analysis revealed that under semi-tandem stance and rigid surface with closed eyes condition, tracers showed lower values for mean power in ML direction at the middlefrequency band than controls. Additionally, under Parkour stance and rigid surface with closed eyes condition, tracers showed lower values for mean power in AP direction at the high-frequency band, and for mean power in ML direction at all frequencies band than controls. Finally, under Parkour stance and soft surface with closed eyes condition, tracers showed lower values for mean power in AP and ML directions at the high-frequency band than controls.

Assuming that the low-frequency band is related to visual control, ${ }^{12,13,26}$ the middlefrequency band is related to vestibular and somatosensory information, ${ }^{12,26}$ and the highfrequency band is related to proprioceptive control and muscle activity, ${ }^{12,26}$ our findings 
indicate that tracers use these sensory inputs to control balance differently when compared to controls.

Furthermore, higher values for mean power at the low-frequency band were related to visual impairments, ${ }^{26}$ higher values for mean power at the middle-frequency band were related to cutaneous and vestibular feedback impairments, ${ }^{26}$ and higher values for mean power at the high-frequency band were related to proprioceptive and muscle activity alterations ${ }^{26}$ or increased muscle activity. ${ }^{24}$

Therefore, these differences between tracers and controls observed in mean power at all frequency bands may reveal some interesting aspects of their postural control's underlying physiological mechanisms. Based on these higher mean power values displayed by controls, it could be suggested that they exhibit less calibrated sensory reweighting than tracers when exposed to similar environmental conditions. Thus, controls would not be able to properly extract and use the most relevant sensory cues in order to control balance. Otherwise, tracers would be able to do that. In this way, the lower values for mean power at all frequency bands observed in tracers could mean that they exhibit more calibrated sensory reweighting. Consequently, tracers also displayed lower amplitudes of the torque required for stabilization, a higher degree of postural stability, and a lower amount of body sway.

All taken together, these results indicate that tracers have better postural control than controls. This is probably due to Parkour training characteristics, which involve numerous movements, and these movements have to be adapted according to the environmental conditions. Considering that decision-making is essential for high-level performance in an unpredictable environment, ${ }^{29}$ the characteristics of Parkour seems to demand a high decision-making performance. Also, to achieve success in decision-making performance, athletes have to enhance action-perception coupling by improving the detection and use of perceptual variables that inform which actions are possible or not to be performed. ${ }^{30}$ In this way, whenever environmental conditions change, sensory inputs must be dynamically reweighed to optimize the control of postural stability ${ }^{3}$, which allows the tracers to perceive the environment properly and then to act in that environment appropriately (action-perception coupling).

In summary, the practice of Parkour provides sensorimotor experiences that improve the underlying physiological and biomechanical mechanisms related to postural control.

Limitations of this study are its exploratory nature and the small sample size. Even though, these preliminary data provide interesting information about the underlying physiological and biomechanical mechanisms related to postural control of tracers. However, further research should be conducted to expand the knowledge of these mechanisms.

\section{CONCLUSION}

This pilot study revealed that tracers have better postural control than controls. Specifically, tracers compared to controls show a lower amount of sway, need less postural commands, and use sensory inputs to control balance differently. Therefore, although current findings are based on data from a small number of subjects, the results suggest that these differences are related to different underlying physiological and biomechanical mechanisms related to postural control. 


\section{REFERENCES}

1. Clark JE. On the problem of motor skill developmet. JOPERD. 2007;78:39-44. doi: 10.1080/07303084.2007.10598023

2. Nashner LM. Analysis of stance posture in humans. In: Towe AL, Luschei ES (eds). Motor coordination (Handbook of behavioural neurology). New York: Plenum Press; 1981:pp.527-564.

3. Horak FB, Macpherson JM. Postural orientation and equilibrium. In: Rowell LB, Shepherd JT (eds). Handbook of physiology: a critical, comprehensive presentation of physiological knowledge and concepts. New York: Oxford University Press; 1996:pp.255-292.

4. Oie KS, Kiemel T, Jeka JJ. Multisensory fusion: simultaneous re-weighting of vision and touch for the control of human posture. Brain Res Cogn Brain Res. 2002;14:164-176. doi: 10.1016/S0926-6410(02)00071-X

5. Schöner G. Dynamic theory of action-perception patterns: the 'moving room' paradigm. Biol Cybern. 1991;64:455-462.

6. Gibson JJ. The senses considered as percep tual systems. Boston: Houghton Mifflin; 1966.

7. Thelen E. Motor development. American Psychologist. 1995;50:79-95.

8. Godoi D, Barela JA. Optical flow structure effects in children's postural control. PLOS ONE. 2016;11:e0158416. doi: 10.1371/journal.pone.0158416

9. Polastri PF, Barela JA. Adaptive visual re-weighting in children's postural control. PLoS ONE. 2013;8:e82215. doi: 10.1371/journal.pone.0082215

10. Allison LK, Kiemel T, Jeka JJ. Sensory-challenge balance exercises improve multisensory reweighting in fall-prone older adults. J Neurol Phys Ther. 2018;42:84-93. doi: 10.1097/NPT.0000000000000214

11. Asseman FB, Caron O, Crémieux J. Are there specific conditions for which expertise in gymnastics could have an effect on postural control and performance? Gait Posture. 2008;27:76-81. doi: 10.1016/j.gaitpost.2007.01.004

12. Nagy E, Toth K, Janositz G, Kovacs G, Feher-Kiss A, Angyan L, et al. Postural control in athletes participating in an ironman triathlon. Eur J Appl Physiol. 2004;92:407-413. doi: 10.1007/s00421-004-1157-7

13. Golomer E, Crémieux J, Dupui P, Isableu B, Ohlmann T. Visual contribution to selfinduced body sway frequencies and visual perception of male professional dancers. Neurosci Lett. 1999;267:189-192. doi: 10.1016/s0304-3940(99)00356-0

14. Stambolieva K, Diafas V, Bachev V, Christova L, Gatev P. Postural stability of canoeing and kayaking young male athletes during quiet stance. Eur J Appl Physiol. 2012;112:18071815. doi: 10.1007/s00421-011-2151-5 
15. Miller JR, Demoiny SG. Parkour: A new extreme sport and a case study. J Foot Ankle Surg. 2008;47:63-65. doi: 10.1053/j.jfas.2007.10.011

16. Cazenave N, Michel $\mathrm{G}$. Conduites à risques et variation de l'estime de soi chez les adolescents: l'exemple du parkour. Annales Médico-Psychologiques. 2008;166:875-881. doi: 10.1016/j.amp.2008.10.026

17. Puddle DL, Maulder PS. Ground reaction forces and loading rates associated with Parkour and traditional drop landing techniques. J Sports Sci Med. 2013;12:122-129.

18. Jabnoun S, Borji R, Sahli S. Postural control of Parkour athletes compared to recreationally active subjects under different sensory manipulations: A pilot study. Eur J Sport Sci. 2018;19:461-470. doi: 10.1080/17461391.2018.1527948

19. Gilchrist $P$, Wheaton $B$. Lifestyle sport, public policy and youth engagement: examining the emergence of parkour. International Journal of Sport Policy and Politics. 2011;3:109-131. doi: 10.1080/19406940.2010.547866

20. Grosprêtre S, Lepers R. Performance characteristics of Parkour practitioners: Who are the traceurs? Eur J Sport Sci. 2016;16:526-535. doi: 10.1080/17461391.2015.1060263

21. Maldonado $G$, Bitard $H$, Watier $B$, Souères $P$. Evidence of dynamic postural control performance in parkour landing. Comput Methods Biomech Biomed Engin. 2015;18:1-3. doi: $10.1080 / 10255842.2015 .1069588$

22. Baratto L, Morasso PG, Re C, Spada G. A new look at posturographic analysis in the clinical context: sway-density versus other parameterization techniques. Motor Control. 2002;6:246-270. doi: 10.1123/mcj.6.3.246

23. Jacono M, Casadio M, Morasso PG, Sanguineti V. The sway-density curve and the underlying postural stabilization process. Motor Control. 2004;8:292-311.

24. Barbosa RC, Vieira MF. Postural control of elderly adults on inclined surfaces. Ann Biomed Eng. 2017;45:726-738. doi: 10.1007/s10439-016-1718-z

25. Vieira TMM, Oliveira LF, Nadal J. An overview of age-related changes in postural control during quiet standing tasks using classical and modern stabilometric descriptors. $J$ Electromyogr Kinesiol. 2009;19:e513-e519. doi: 10.1016/j.jelekin.2008.10.007

26. Vieira MF, Avelar IS, Silva MS, Soares V, Lobo Da Costa PH. Effects of four days hiking on postural control. PLOS ONE. 2015;10:e0123214. doi: 10.1371/journal.pone.0123214

27. Pena GM, Pavão SL, Oliveira MF, P., Godoi D, Decampos AC, Rocha NaF. Dual-task effects on postural sway during sit-to-stand movement in children with Down syndrome. $J$ Intellect Disabil Res. 2019;63:576-586. doi: 10.1111/jir.12599

28. Cohen J. Statistical power analysis for the behavioral sciences. 2nd ed. ed. New Jersey, USA: Lawrence Erlbaum Associates; 1988.

29. Gil-Arias A, Moreno MP, García-Mas A, Moreno A, García-González L, Villar F. Reasoning and action: Implementation of a decision-making program in sport. Span. J. Psychol. 2016;19:1-9. doi: 10.1017/sjp.2016.58 
Brazilian Journal of Motor Behavior

\section{Research Article}

30. Travassos B, Araújo D, Davids K, Vilar L, Esteves P, Vanda C. Informational constraints shape emergent functional behaviours during performance of interceptive actions in team sports. Psychol Sport Exerc. 2012;13:216-223. doi: 10.1016/j.psychsport.2011.11.009

\section{ACKNOWLEDGEMENTS}

The authors are grateful to all participants who volunteered to take part in this study.

Citation: Veneroso AFV, Segundo PW, Godoi D. Underlying physiological and biomechanical mechanisms related to postural control of parkour practitioners: a pilot study. BJMB. 2021. 15(2): 65-78.

Editors: Dr Fabio Augusto Barbieri - São Paulo State University (UNESP), Bauru, SP, Brazil; Dr José Angelo Barela São Paulo State University (UNESP), Rio Claro, SP, Brazil; Dr Natalia Madalena Rinaldi - Federal University of Espírito Santo (UFES), Vitória, ES, Brazil.

Copyright: $\odot 2021$ Veneroso, Segundo and Godoi and BJMB. This is an open-access article distributed under the terms of the Creative Commons Attribution-Non Commercial-No Derivatives 4.0 International License which permits unrestricted use, distribution, and reproduction in any medium, provided the original author and source are credited. Funding: This research did not receive any specific grant from funding agencies in the public, commercial, or not-forprofit sectors.

Competing interests: The authors have declared that no competing interests exist.

DOI: https://doi.org/10.20338/bjmb.v15i2.207 